\title{
DifFERENTIATED SERVICES ENSURING QOS ON INTERNET
}

\author{
Pawansupreet Kaur ${ }^{1}$, Monika Sachdeva ${ }^{2}$ and Gurjeet Kaur ${ }^{3}$ \\ ${ }^{1}$ Department of Computer Engineering, SBS State Technical Campus, Ferozpur, Punjab \\ Meens399@gmail.com \\ ${ }^{2}$ Department of CSE, Associate Proff at SBS State Technical Campus, Ferozpur, Punjab \\ Monika.sal@rediffmail.com \\ ${ }^{3}$ Department of Computer Engineering, SBS State Technical Campus, Ferozpur, Punjab \\ Gurjeetrandhawa4@gmail.com
}

\begin{abstract}
Differentiated Services is the most advanced method for traffic management which provides QoS. QoS is essential for today's internet. QoS represents set of techniques necessary to manage throughput, packet loss and delay. Traffic Conditioners or Policers are the most important components of differentiated services. In this research, different Traffic Conditioners or Policers are compared on the basis of QoS parameters (Performance metrics) which are throughput, packet loss and delay to provide QoS on internet. Traffic classification and traffic conditioning are important functions of differentiated services also known as admission control which are governed by service level agreement (SLA). Traffic classification and traffic conditioning is done by traffic conditioners at edge routers. Goal of this research is to choose the best Traffic Conditioner to apply from source to destination pair because only one traffic conditioner can be applied from source to destination so that QoS can be guaranteed.
\end{abstract}

\section{KEYWORDS}

Differentiated Services, Performance Metrics, QoS, Traffic Conditioners or Policers

\section{INTRODUCTION}

The Internet was designed as a best effort network for transporting computer-to-computer traffic. However, as the footprint of the Internet grew, a wide variety of applications emerged. The growth in the diversity and volume of Internet applications made it essential to discover and implement new techniques that support different levels of service for different classes of traffic. These techniques are collectively referred to as Quality of Services (QOS) techniques. Differentiated Services [1] was introduced in the late 1990s in response to the need for a simple, yet effective QOS mechanism suitable for implementation on the Internet. Differentiated services has been proposed as an efficient and scalable traffic management mechanism way to ensure internet QOS. QoS is the classification of packets for the purpose of treating certain classes or flows of packets in a particular way as compared to the other packets. Various solutions have been proposed to guarantee QoS. In 1997 IETF proposed an architecture that is Differentiated Services architecture. The Differentiated Services (Diff Ser) network architecture attempts to provide these QoS guarantees in the most scalable and least complex manner. The Diff Ser approach is based on a set of simple mechanisms that treat packets differently according to the marking of the DS field in the IP header [2]. In differentiated services, complexity is pushed at edge routers with core remains with simple functionality. Packets are marked with different

DOI : 10.5121/ijcseit.2012.2504 
Traffic Conditioners or Policers at edge routers to receive particular behaviour. My research work focus on these traffic conditioners. Five different traffic conditioners are selected for the comparison. These Traffic Conditioners or Policers are compared on the basis of different Performance metrics. Differentiated Services are implemented with NS2 Simulator. One of the Differentiated Services packet forwarding treatments [3], Assured Forwarding PHB [4] is implemented in this research work. The Differentiated Services Assured Forwarding (AF) PerHop Behaviour Group defines a differentiated forwarding of packets in four independent classes, having three levels of drop precedence.

\section{Traffic Conditioners And Performance Metrics}

A traffic conditioner may contain meters, markers, shapers and droppers for traffic conditioning functions. The conditioner may re-mark a traffic stream or may discard or shape packet to alter the temporal characteristics of the stream and bring it into compliance with a traffic profile specified by the network administrator.

\subsection{Traffic Conditioners}

Traffic Conditioners are most important components of differentiated services. Packets are marked with different Traffic Conditioners or Policers at edge routers to receive particular behaviour which are (1) TSW2CM (2) TSW3CM (3) Token Bucket (4) srTCM (5) trTCM

\subsubsection{TSW2CM (Time Sliding Window Two Colour Marker)}

TSW2CM uses a CIR and two drop precedence. The lower one is used probabilistically when CIR is exceeded.

\subsubsection{TSW3CM (Time Sliding Window Three Colour Marker)}

TSW3CM [5] uses a CIR, and PIR and three drop precedence. When the measured traffic is below the CIR, packets are marked with low drop precedence. If the measured traffic exceeds its CIR, packets are marked with medium drop precedence. If the traffic exceeds its PIR, packets are marked with highest drop precedence.

\subsubsection{Token Bucket}

Token Bucket [6] uses a CIR and CBS, and two drop precedence. The token bucket marker (TBM) meters a traffic stream based on two parameters: CIR (committed information rate) and CBS (committed burst size). The meter is specified in terms of a token bucket with size CBS and token generation rate CIR. Tokens are generated at a rate equal to the CIR and are added to the token bucket provided it is not full.

\subsection{4. srTCM (Single Rate Three Colour Marker)}

srTCM [7] uses a CIR, CBS and EBS to choose from three drop precedence.

\subsection{5. trTCM (Two Rate Three Colour Marker)}

trTCM [8] uses a CIR, CBS, EBS, and PBS to choose from three drop precedence. 


\subsection{Performance Metrics}

\subsubsection{Throughput}

Throughput is a generic term used to describe the capacity of the system to transfer data. Throughput is nothing but the bandwidth of the transmission channel. Throughput is the rate at which network sends or receives data. Throughput is calculated using packets received divided by packets forwarded.

Throughput $=$ Packets received $/$ Packets forwarded

\subsubsection{End To End Delay}

Delay is the amount of time that it takes for a packet to be transmitted from one point in network to another point in a network. It refers to the time taken for a packet to be transmitted across a network from source to destination.

\subsubsection{Packet Loss}

Packet loss is where network traffic fails to reach its destination in a timely manner.

Packet Lost $=$ amount of packets received - amount of packets forwarded

\section{SiMULATION ENVIRONMENT}

In this research paper, we are comparing different traffic conditioners for the TCP type traffic. Differentiated Services are implemented in NS2 Simulator. Different traffic conditioners are configured with same configuration parameters for better analysis. First, we have created a topology. Then traffic pattern has been defined for that topology. Second Traffic Conditioners are configured on ingress edge routers. Packet scheduling mechanism FIFO is implemented at egress edge routers. RED with IN/OUT (RIO), a differentiated dropping algorithm is used in interior routers. Our simulation is done with TCP type traffic for 20 source nodes which are generating traffic at same rate. Each source node is connected to a corresponding edge node where traffic is marked according to the parameters that will be specified. The edge routers are connected to a bottleneck core router and then through another edge router, to a destination node. Links between the edge nodes and corresponding source nodes have delays of $10 \mu \mathrm{sec}$ and $6 \mathrm{Mbps}$ bandwidth. Links between the edge nodes and corresponding destination nodes have delays of $10 \mu \mathrm{sec}$ and $10 \mathrm{Mbps}$ bandwidth. Links between the core node and edge nodes that are attached to the sources have delays of $0.1 \mathrm{msec}$ and $6 \mathrm{Mbps}$ bandwidth. Links between the core node and edge node that are attached to the destination has delays of $1 \mathrm{msec}$ and 10Mbps bandwidth. Queues can be built at the bottleneck router, i.e. at the link between the core node and the edge node that connects to the destination. We choose its size to be of 100 packets. . In the bottleneck queue at the core node, a multi-RED queue management is used with a RIO-D version. Figures1 represents the network topology which is used for simulating differentiated services. There are 20 source nodes which are generating traffic at the same rate. 


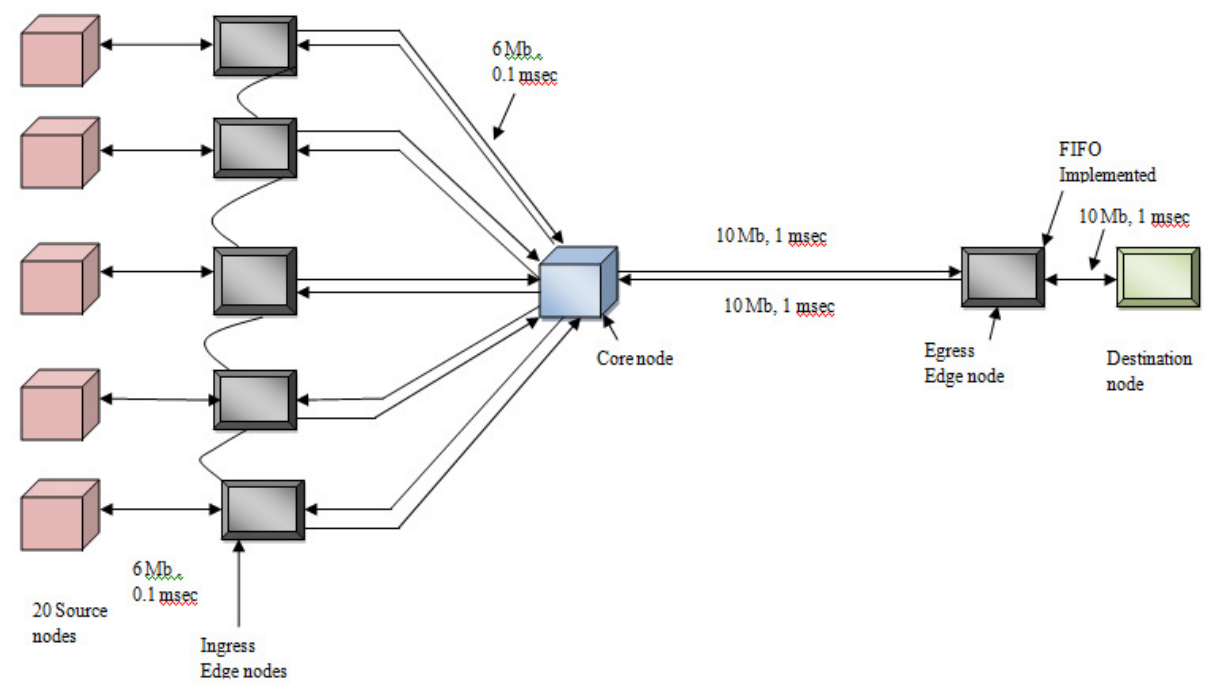

Fig 1: Network topology

\section{Simulation RESUltS}

In this section, we present the simulation results with different traffic conditioners that we have described in the previous section. The simulation is done with discrete event simulation namely ns2 version 2.34. Models in ns are created by defining nodes and connecting them with links to form some network topology

\subsection{Throughput}

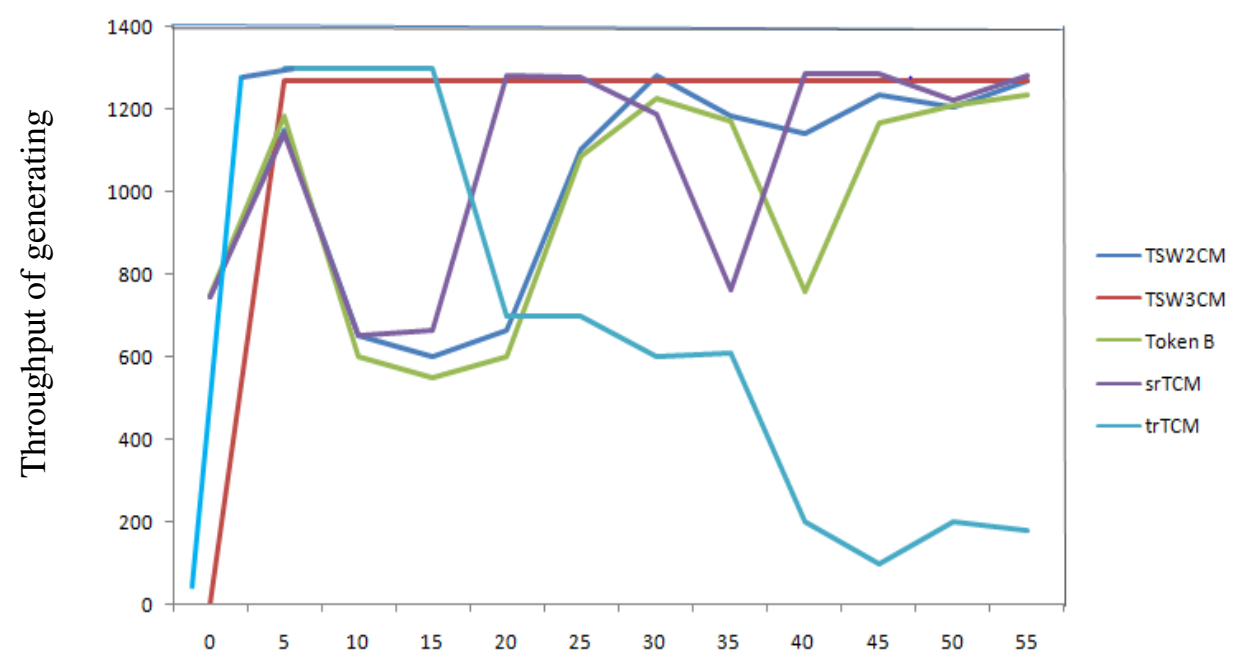

Simulation time (sec)

Fig 2: Throughput 
The minimum assured throughput is given according to the negotiated profile with the client. TSW2CM, Token Bucket and srTCM bears almost same variation. In case of TSW3CM throughput is high at start, then it becomes constant. In case of trTCM, throughput is high at start and after that it starts decreasing because throughput increases as long as there are available resources and decreases when congestion occurs. Secondly source is generating traffic at more rate than committed rate. So more packets were marked as out-of-profile i.e when congestion occurs these can be dropped. In case of srTCM, throughput bears different variations, at some point it is high and then suddenly decreases and then again starts increasing and at the end throughput is high because in this case we have more options to mark the packets so We can assign different drop precedences to different packets. srTCM gives high throughput.

\subsection{Packet Loss}

Packet Statistics are shown in the tables.

Table 1. TSW2CM

\begin{tabular}{|c|c|c|c|c|}
\hline CP & TotPkts & TxPkts & Ldrops & edrops \\
\hline All & 60593 & 58892 & 140 & 1561 \\
\hline 10 & 14633 & 14633 & 0 & 0 \\
\hline 11 & 45960 & 44259 & 140 & 1561 \\
\hline
\end{tabular}

Table 2. TSW3CM

\begin{tabular}{|c|c|c|c|c|}
\hline CP & TotPkts & TxPkts & Ldrops & Edrops \\
\hline All & 60449 & 58904 & 125 & 1420 \\
\hline 10 & 14751 & 14751 & 0 & 0 \\
\hline 11 & 39573 & 44259 & 125 & 1420 \\
\hline 12 & 6125 & 6125 & 0 & 0 \\
\hline
\end{tabular}

Table 3. Token Bucket

\begin{tabular}{|c|c|c|c|c|}
\hline CP & TotPkts & TxPkts & Ldrops & edrops \\
\hline All & 60433 & 58653 & 136 & 1644 \\
\hline 10 & 14630 & 14630 & 0 & 0 \\
\hline 11 & 45803 & 44023 & 136 & 1644 \\
\hline
\end{tabular}

Table 4. srTCM

\begin{tabular}{|c|l|l|c|c|}
\hline CP & TotPkts & TxPkts & Ldrops & edrops \\
\hline All & 59603 & 58734 & 153 & 716 \\
\hline 10 & 4027 & 4014 & 13 & 0 \\
\hline 11 & 31471 & 30683 & 72 & 716 \\
\hline 12 & 24105 & 24037 & 68 & 0 \\
\hline
\end{tabular}


Table 5. $\operatorname{trTCM}$

\begin{tabular}{|c|l|l|c|c|}
\hline CP & TotPkts & TxPkts & Ldrops & edrops \\
\hline All & 60495 & 58521 & 181 & 1793 \\
\hline 10 & 5332 & 5332 & 0 & 0 \\
\hline 11 & 55163 & 53189 & 181 & 1793 \\
\hline
\end{tabular}

CP stands for code point, TotPkts are amount of total forwarded packets and TxPkts are amount of total received packets. Ldrops are link drops and edrops are those drops which are due to early detection. Packet loss is calculated using formula (Packet Loss $=$ amount of packets received amount of packets forwarded). In TSW2CM, total Packet Loss is 1701. Link drops are 140 and edge drops i.e. drop because of early detection are 1561. Edrops are more than ldrops because more traffic handling is done at edge routers and if packets exceed than its SLA or TCA then they are dropped at edge routers. Packets marked with code point 10 were marked with low drop precedence so packet loss is less than packets marked with code point 11 which is marked as high drop precedence. It means packets marked with high drop precedence are discarded first than packets marked with low drop precedence in case of congestion. In case of TSW3CM, total packet loss is 1439 . In case of Token Bucket, total packet loss is 1780. In case of srTCM, total packet loss is 869 .. In case of trTCM, total packet loss is 1780 . srTCM drops much less packets as compared to other policers.

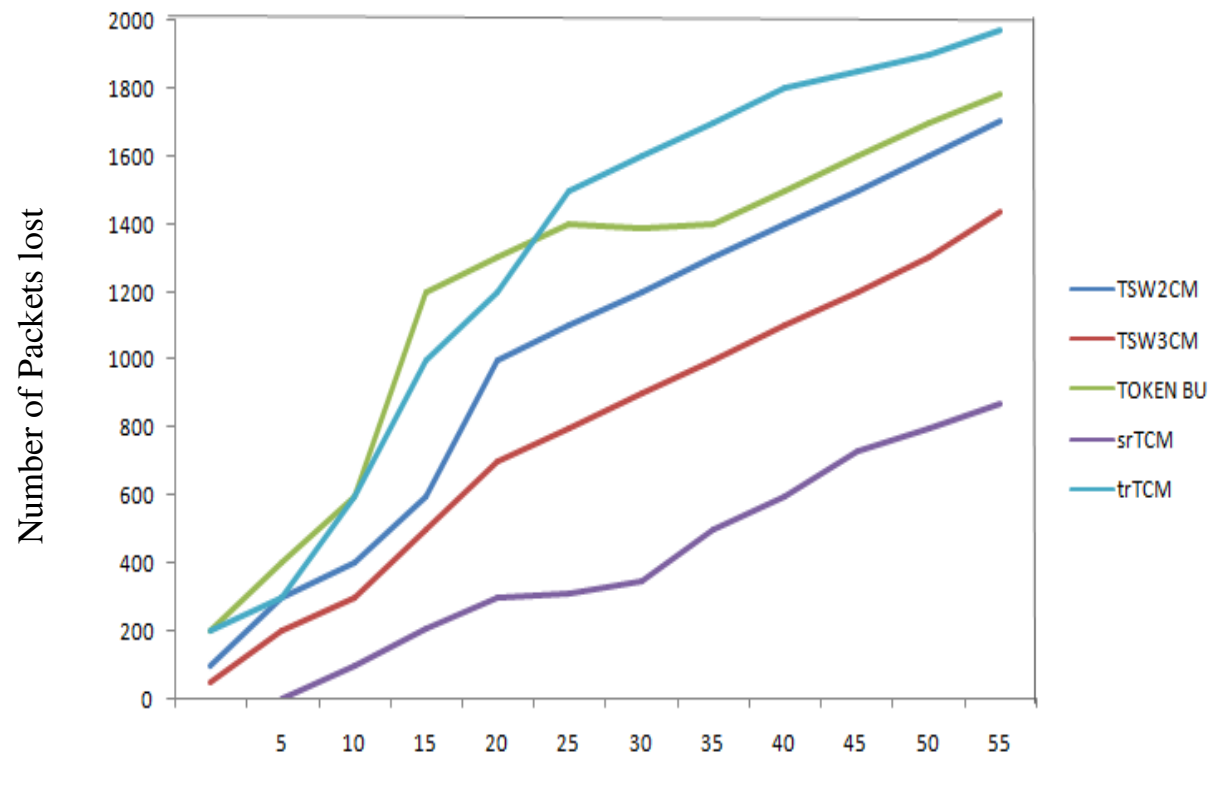

Simulation time

Fig 3: Packet Loss

\subsection{End To End Delay}

Delay is the amount of time that it takes for a packet to be transmitted from one point in network to another point in a network. End to End delay for different policers is shown in table. For real time application or critical applications end to end delay should be less. Lesser the end to end delay, more will the Quality. 


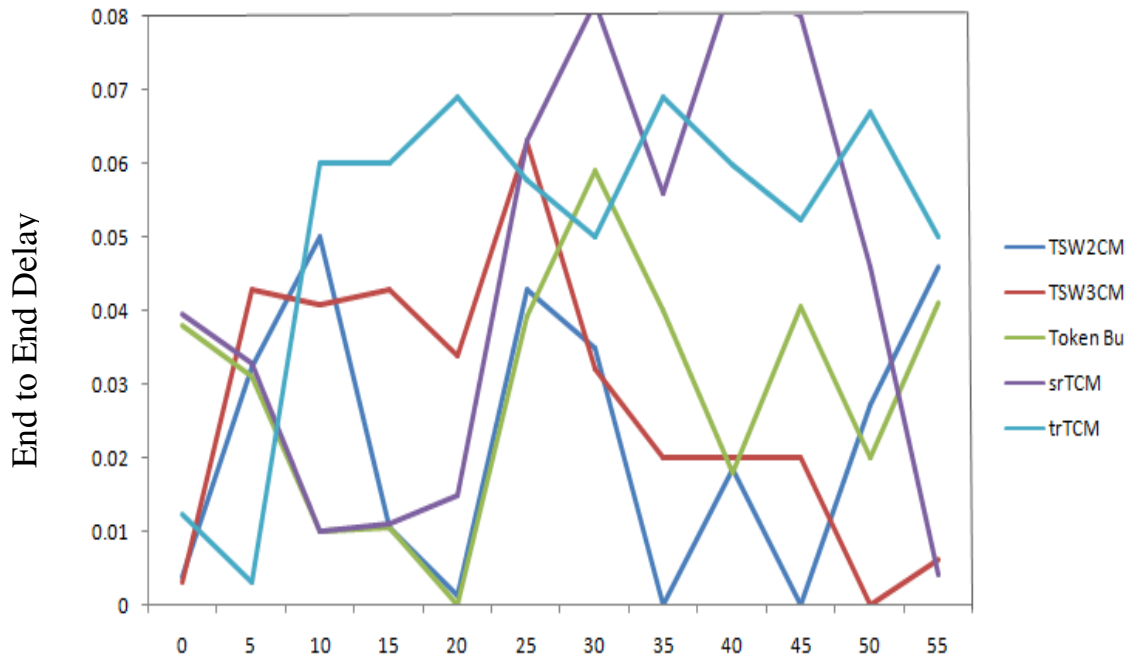

Simulation time

Figure 4. End to End delay

End to End delay for all Traffic Conditioners is shown in the graph corresponding to the simulation time. End to End delay bears large variation in the graph, somewhere it is more and somewhere it is less. End to end delay for TSW2CM is initially 0.05 then it drops to 0.01 and then again more and at the end to end delay is 0.046. In case of TSW3CM, end to end delay initially is 0.043 and at the end it is 0.00608 . End to End delay for TSW3CM is much less as commpared to TSW2CM. That means among TSW group, TSW3CM is better than TSW2CM when comapred according to end to end delay metric.In case of Token Bucket, end to end delay initially is 0.038 , and at the end it is 0.041 . In cae of srTCM, end to end delay initially is 0.0395 , and at the end it is 0.0403. In case of trTCM, Initially end to end delay is 0.0123 and at the end it is 0.05. Among Token Bucket group srTCM has less end to end delay as compared to Token Bucket and trTCM. And among TSW3CM and srTCM , srTCM provides less end to end delay.

\subsection{Combination and Overall Performance}

Table 6. Overall Performance

\begin{tabular}{|c|c|c|c|}
\hline Traffic Conditioners & Packet Loss & End to End delay & Throughput \\
\hline TSW2CM & 1701 & 0.00811 & 0.971 \\
\hline TSW3CM & 1439 & 0.00810 & 0.976 \\
\hline Token Bicket & 1780 & 0.00848 & 0.970 \\
\hline srTCM & 869 & 0.00114 & 0.98 \\
\hline trTCM & 1974 & 0.0123 & 0.96 \\
\hline
\end{tabular}




\section{Conclusions}

All traffic conditioners are different from each other through target rate or configuration parameters by which traffic can be marked differently. When performance was measured in terms of target rate parameters, there was no convincing evidence to suggest that the choice of traffic conditioner affects a source's achieved rate. Therefore, if a network designer is only interested in the relative target rate performance of the traffic conditioners, then he is free to choose any one of the traffic conditioners. When Traffic Conditioners are compared based on throughput then all Conditioners gives almost equal throughput because all the conditioners are configured with same parameters as we are implementing Assured Services, Assured Services are guaranteed to provide minimum throughput in case of congestion also. srTCM gives high throughput as compared to others. When Traffic Conditioners are compared according to packet loss, then among TSW group, TSW3CM drops fewer packets and if compared among Token bucket group then srTCM drops fewer packets. When Traffic Conditioners are compared based on End- to-End Delay then token bucket based srTCM experiences less delay and TSW3CM among TSW group bears less delay. TSW3CM and srTCM gives better performance then other policers. If to choose among TSW3CM and srTCM then srTCM gives better performance and provide QoS requirements i.e. Less delay, less packet loss and more throughputs. srTCM is best to choose.

\section{REFERENCES}

[1] Blake, S. Black, D. Carlson, M. Davies, E. Wang, Z. and Weiss, WS. (2008) "An architecture for Differentiated Services”, RFC 2475.

[2] Nichols, K. (1998) "Definition of Differentiated Services Field (DS Field) in the IPv4 and IPv6 Headers", RFC 2474.

[3] Brim, S. Carpenter, B. and Faucheur, F.le ( 2000) "Per Hop Behavior Codes", RFC 2836.

[4] Heinanen, J. Baker, F. Weis, and W. Wroclawski, J. (1999) "Assured forwarding PHB Group".

[5] Fang, W. Seddigh, N. and Nandy, B. (2000) "Time Sliding Window Three Color Marker (TSW3CM)", RFC 2859.

[6] Heinanen, J. and Guerin, R. (2000) “A Single Rate Three Color Marker”, RFC 2697.

[7] Stallings, W. (1999) "High Speed Networks: TCP/IP and ATM Design Principles", Prentice Hall Englewood cliffs, NZ.

\section{Authors}

Pawansupreet Kaur was born in Ferozepur, Punjab, on July 23, 1989. Pawansupreet Kaur received her Bachelor of Technology and Engineering Degree from Adesh Institute of Engineering and Technology, Punjab, India. Currently she is doing Masters in Computer Science and Engineering from Shaheed Bhagat Singh State Technical Campus, Ferozepur, Punjab, India. Her Research Interest are in the areas of Networking and Mobile Telecommunication. Miss Pawansupreet Kaur is member of UACEE.

Monika Sachdeva has done Btech in computer Science Engineering from National Institute of Technology (NIT), Jalandhar in 1997. She has done her MS software systems from BITS pilani in 2002. She has done her Phd in Computer Science and Engineering from Guru Nanak Dev University, Amritsar, Punjab, India in 2012.

Gurjeet Kaur was born in Pehowa, Haryana, on December 1, 1986.Gurjeet Kaur received her Bachelor of Technology degree in Computer Science and Engineering from Adesh Institute Of Engineering \& Technology, Punjab,India. Currently she is doing Master's in Computer Science \& Engineering from Shaheed Bhagat Singh State Technical campus, Ferozepur, Punjab, India. Her research interests are in the area of Telecommunication Networks (GSM Security), Location Based Services. Miss Gurjeet

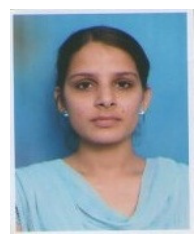
Kaur is member of UACEE and SDIWC.

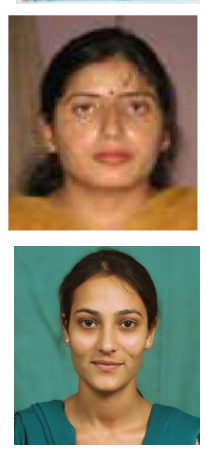

